\title{
Green Synthesized Cobalt Nano Particles for using as a Good Candidate for Sensing Organic Compounds
}

\author{
S. O. Ranaei Siadat* \\ Protein Research Center, Shahid Beheshti University, GC, Tehran, Iran
}

\begin{abstract}
In this work electrochemical oxidation of Ascorbic acid (AA) on the surface of Cobalt nano particle modified carbon paste electrode (CoNPsMCPE) was studied in alkaline media. CoNPs were green synthesized using Piper longum and a mixture of $5 \%(\mathrm{w} / \mathrm{w})$ of it were made with carbon paste. CoNPs showed good electrocatalytic activity in alkaline media. Cyclic voltammetry $(\mathrm{CV})$ and chronoamperometry $(\mathrm{CA})$ were used to study the electrochemical performance of CoNPsMCPE. The number of monolayers on the surface of electrode was calculated as $1.08 \times 10^{9} \mathrm{~mol} \mathrm{~cm}^{-2}$ that is equal to that of metal Cobalt electrode. Diffusion coefficient of AA was determined using CA analysis which was equal to $1.5 \times 10^{-6} \mathrm{~cm}^{2} \mathrm{~s}^{-1}$.
\end{abstract}

Keywords : Green synthesis, Cobalt nanoparticles, Ascorbic acid, Carbon paste electrode, Alkaline media.

Received August 4, 2015 : Accepted September 9, 2015

\section{Introduction}

One of the great areas of electrochemical researches is chemically modified electrodes using as electrochemical sensors. There are two major methods for electrode modification; surface and bulk modification. Surface modification is an effective method including adsorption, covalent bonding, attachment of polymer films etc. [1-3]. One of the major parameters in producing modified electrodes is the nature of working electrode. Carbon paste electrode (CPE) due to ease of Preparation and capability to obtain a new reproducible surface is selected by researchers. Furthermore, having porous surface, low cost and low residual current are other advantages of CPE than other solid electrodes [4,5]. Recently the materials that are immobilized onto the electrode surface and their ability for fast electron transferring are under focused [6,7]. Transition metals with their good electrochemical capabilities as active materials are reported many times in literature [8-10]. $\mathrm{Ni}$ [3], $\mathrm{Cu}$ [11], $\mathrm{Au} \mathrm{[12],} \mathrm{Ru} \mathrm{[13]} \mathrm{and} \mathrm{Co}$ are some transition metals that are reported for use in electrochemical sensors. Cobalt nano particle based materials which can flip-flop between various valence states under the effect of external electric fields in one hand and a potential reducing agent on the other hand are particularly interested in this regard. Various methods have been reported for synthesis of cobalt electrodes [14-16], but the synthesis of cobalt nanoparticles are very sensitive because many parameters effect on its physioelectrochemical properties [17]. Various methods such as chemical and physical synthetic procedures exist for production of nano particles that have limitations such as using toxic, expensive chemicals or devices.

*Corresponding author. Tel.: +98 (21) 22431782

E-mail address: ranaeisiadat@hotmail.com,o_ranaei@sbu.ac.ir

Open Access DOI: http://dx.doi.org/10.5229/JECST.2015.6.4.111

This is an Open Access article distributed under the terms of the Creative Commons Attribution Non-Commercial License (http://creativ ecommons.org/licenses/by-nc/3.0/) which permits unrestricted non-commercial use, distribution, and reproduction in any medium, provided the original work is properly cited. 
However, plant extract materials have been used for synthesis of nano particles which could be advantageous over chemical and physical methods. Cost effectiveness, simple sampling and environmental friendly are some advantages of green synthesis of nanoparticles [18-20]. Ascorbic acid is a powerful antioxidant naturally present in many foods, especially fruits and vegetables, and plays an important role in the prevention of infectious diseases. AA is frequently used in food industry as an antioxidant to prevent undesirable changes in color, taste and odor. It is of great interest to have a rapid and sensitive method for routine and reliable determination of AA. In the present study, the synthesis of Cobalt nano particles, and modification of carbon paste electrode using these nano particles for electro-oxidation of Ascorbic acid are presented. We modified the surface of CPE with Co/NPs and then studied the physioelectrochmical oxidation of AA in alkaline media.

\section{Experimental}

\section{The cell and instruments}

All electrochemical studies were carried out in a conventional three electrode cell powered by an EG\&G model 273 potentiostat/galvanostat. The system is run by a PC through M270 interface. A dual $\mathrm{Ag}-\mathrm{AgCl}$ saturated $\mathrm{KCl}$ and a $\mathrm{Pt}$ wire electrode were used as reference and counter electrodes, respectively. All studies were carried out at room temperature.

\section{CoNPs preparation}

CoNPs were synthesized using green method as described in [21]. $50 \mathrm{~g}$ of dried powdered fruits of Piper longum was added to $300 \mathrm{~mL}$ of $30 \%$ methanol solution in a $500 \mathrm{~mL}$ flask and well mixed. It was heated at $70^{\circ} \mathrm{C}$ under vigorous stirring for $30 \mathrm{~min}$ and then was centrifuged and extracted.

In a typical synthesis of CoNPs, $15 \mathrm{~mL}$ of the extract of the plant fruits was added drop wise to $50 \mathrm{~mL}$ of $0.003 \mathrm{M}$ aqueous solution of $\mathrm{CoCl}_{2}$ with constant stirring at $80^{\circ} \mathrm{C}$. Reduction of Co ions $\left(\mathrm{Co}^{\mathrm{II}}\right)$ to $\mathrm{Co}^{0}$ was completed after $30 \mathrm{~min}$ during which, changing the color of the reaction mixtures indicated the formation of Co nanoparticles. Then the colored solution of Co nanoparticles was centrifuged at 7000 rpm for $40 \mathrm{~min}$ to absolute precipitation of Co NPs.

\section{Preparation of working electrode}

Mixtures of CoNPs and graphite powder with ratio 5:95 were added to pure ethanol. Then the mixture heated and stirred by a magnet to evaporate the ethanol. Then it was mixed with mineral oil $(75: 25)$ and was blended by hand mixing with a mortar and pestle for preparation of carbon paste. The resulting paste was then inserted in the bottom of a glass tube with the area of $0.07 \mathrm{~cm}^{2}$. The electrical connection was implemented by a copper wire lead fitted into the glass tube. A fresh electrode surface was generated rapidly by extruding a small plug of the paste with a stainless-still rod and smoothing the resulting surface on white paper until a smooth shiny surface was observed. All the Materials in this work were of analytical grade, purchased form Sigma-Aldrich Co. and were used without any purification.

\section{Results and Discussion}

Fig. 1 presents cyclic voltammograms (CVs) of $\mathrm{CPE}$ and CoNPsMCPE in $\mathrm{NaOH} 0.1 \mathrm{M}$ at the scan rate of $100 \mathrm{mV} / \mathrm{s}$. As can be seen, in the absence of CoNPs there is no peak in CV of CPE, whereas by the addition of CoNPs a redox peak can be observed which is related to $\mathrm{Co}(\mathrm{III}) / \mathrm{Co}(\mathrm{IV})$. Because of the magnitude of nano particles' peak and the capacitive behavior of CPE, the redox peak related to $\mathrm{Co}$ (II)/ Co(III) charge transfer may has been disappeared [7,22].

Fig. 2 presents $\mathrm{CVs}$ of modified electrode in $\mathrm{NaOH}$ $0.1 \mathrm{M}$ at various scan rates. As can be seen by increas-

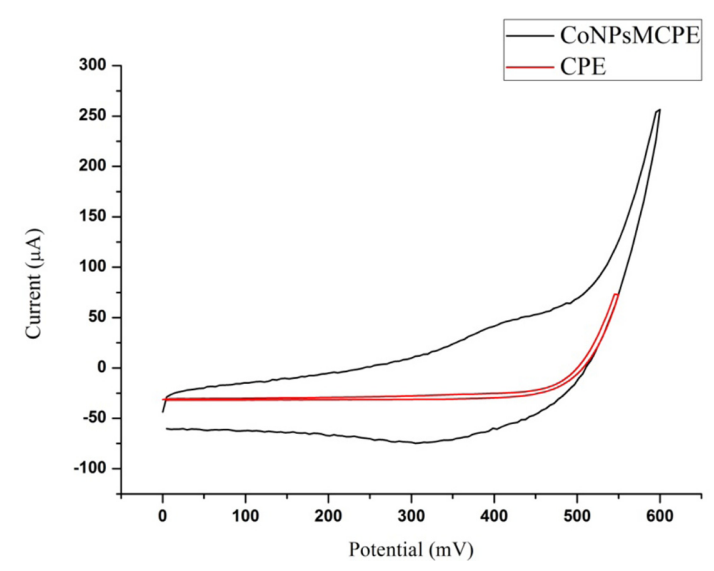

Fig. 1. Cyclic voltammograms of CPE and CoNPsMCPE in $\mathrm{NaOH} 0.1 \mathrm{M}$ at the scan rate of $100 \mathrm{mV} / \mathrm{s}$. 
ing the sweep rate, the anodic peaks shifted to positive potentials whereas the cathodic ones to negative potentials.

For indicating the electrochemical activity of the surface redox couples, the plot of anodic and cathodic current peaks against potential sweep rate at low values was used. The surface coverage of redox species calculated as follows[23]:

$$
I_{p}=\left(\frac{n^{2} F^{2}}{4 R T}\right) v A \Gamma^{*}
$$

where $\Gamma^{*}$ is the surface coverage of the redox species and $v$ is the potential sweep rate taking average of both cathodic and anodic results. $\Gamma^{*}$ was calculated around $1.08 \times 10^{9} \mathrm{~mol} \mathrm{~cm} \mathrm{~cm}^{-2}$ which is almost equal to that of other reports [24].

Fig. 3 presents the CVs of CoNPsMCPE in the presence of various concentrations of $\mathrm{AA}$ in $0.1 \mathrm{M}$

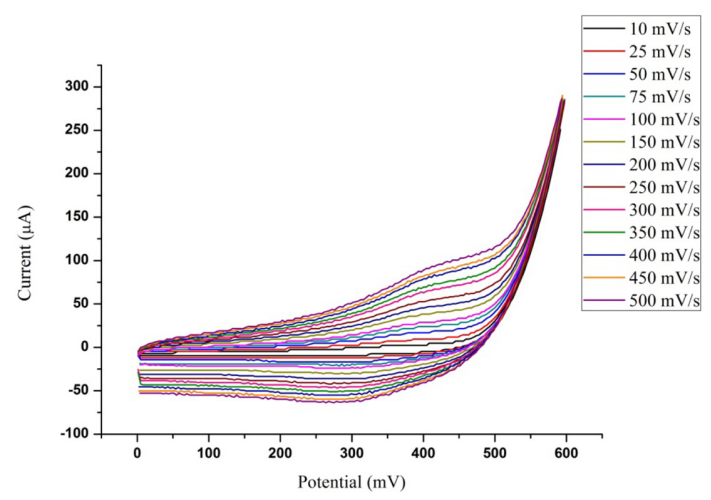

Fig. 2. $\mathrm{CVs}$ of $\mathrm{CoNPs} / \mathrm{CPE}$ in $\mathrm{NaOH} 0.1 \mathrm{M}$ at the various scan rates.

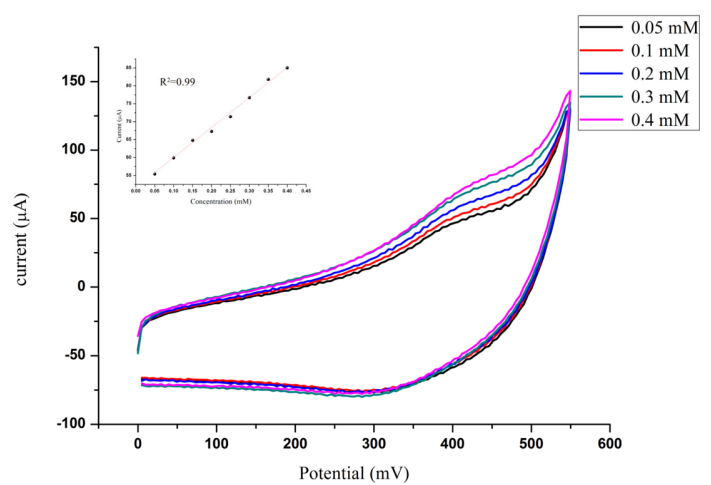

Fig. 3. CVs of CoNPsMCPE in the presence the various concentrations of $\mathrm{AA}$ in $0.1 \mathrm{M} \mathrm{NaOH}$ at the scan rate of $50 \mathrm{mV} / \mathrm{s}$.
$\mathrm{NaOH}$ at the scan rate of $50 \mathrm{mV} / \mathrm{s}$. As can be seen, by increasing the concentration of AA the current of anodic sweep enhances. This results show that the modified electrode has good activity for oxidation of AA in alkaline media. Fig. 3. (inset) shows the calibration curve for AA concentration. As can be seen, the modified electrode has a linear response to AA concentration.

In order to investigate the electrocatalytic activity of CoNPsMCPE, CVs of the electrode at $0.1 \mathrm{M}$ $\mathrm{NaOH}$ solution containing $0.4 \mathrm{mM}$ AA were recorded (Fig. 4). The pattern shows that AA electrooxidation has fast kinetic properties in which, by increasing the sweep rate the anodic peak of AA oxidation and Co peak do not separate. Fig. 4 (inset) is the diagram of $\mathrm{I}_{\mathrm{p}}$ versus the square root of scan rate. The diagram shows that the electrooxidation of AA occurres under mass transfer controlled process. To study some physioelectrochemical properties of the modified electrode, double potential step chronoamperometry was used. Fig. 5 shows the double step chronoamperograms of the modified electrode at various concentrations of AA. The first potential step is pointed at $440 \mathrm{mV}$ that was derived from oxidation potential of AA and the second step pointed at $100 \mathrm{mV}$ that is far from the potential of AA oxidation. At first step, by increasing the concentration of AA the current enhanced, but all diagrams at second step are the same implying that AA electrooxidation on the surface of CoNPsMCPE is irreversible. In addition, chronoamperometry was used to evaluate chemical reaction rate between AA and modified layer (k) according to:

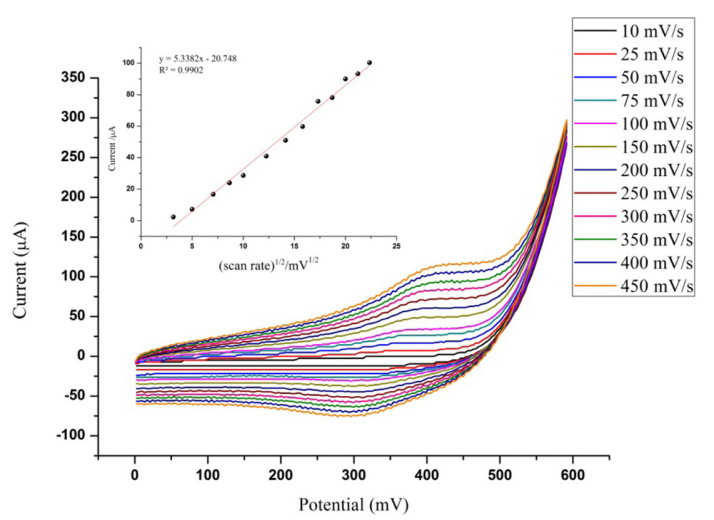

Fig. 4. CVs of CoNPsMCPE in the presence of $4 \mathrm{mM}$ AA at various scan rates. 


$$
I_{c} / I_{I}=\gamma^{1 / 2}\left[\pi^{1 / 2} \operatorname{erf}\left(\gamma^{1 / 2}\right)+(\exp )(-\gamma) / \gamma^{1 / 2}\right]
$$

where $I_{C}$ and $I_{L}$ are currents in the presence and absence of AA respectively, $\gamma=\mathrm{kct}$ is the argument of the error function, $\mathrm{k}$ is the catalytic rate constant, $\mathrm{C}$ is bulk concentration of AA and $t$ is the elapsed time. In the cases where $\tilde{a}>1.5, \operatorname{erf}\left(\tilde{a}^{1 / 2}\right)$ is almost equal to unity and the above equation can be reduced to [25]:

$$
I_{c} / I_{I}=\gamma^{1 / 2} \pi^{1 / 2}=\gamma^{1 / 2}(\mathrm{kct})^{1 / 2}
$$

From the slope of the $\mathrm{I}_{\mathrm{C}} / \mathrm{I}_{\mathrm{L}}$ versus $\mathrm{t}^{1 / 2}$ plot, presented in Fig. 6 (left), the mean value of $\mathrm{k}$ for the concentration range of 0.1 to $0.5 \mathrm{M}$ of $\mathrm{AA}$ was obtained as $1.4 \times 10^{6} \mathrm{~cm}^{3} \mathrm{~mol}^{-1} \mathrm{~s}^{-1}$. Furthermore with this method we can obtain the diffusion coefficient according to:

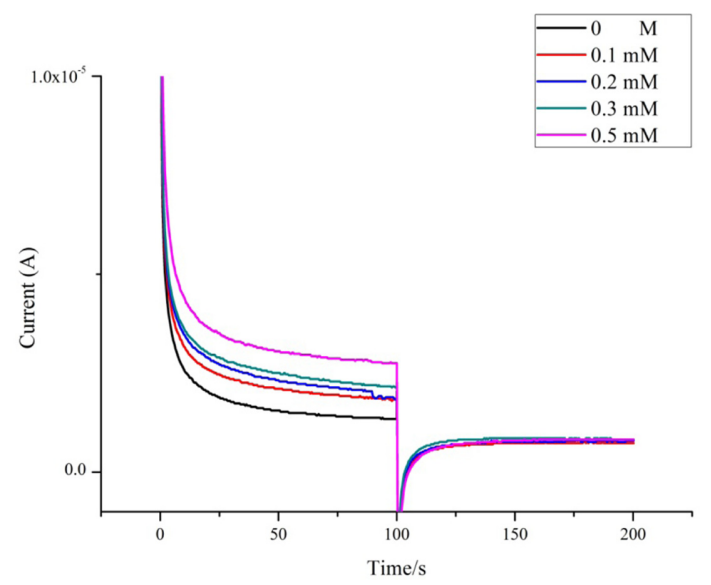

Fig. 5. Double step potential chronoampermograms of CoNPsMCPE in $0.1 \mathrm{M} \mathrm{NaOH}$ at various concentrations of AA. The first step pointed at $440 \mathrm{mV}$ and the second one at $100 \mathrm{mV}$.

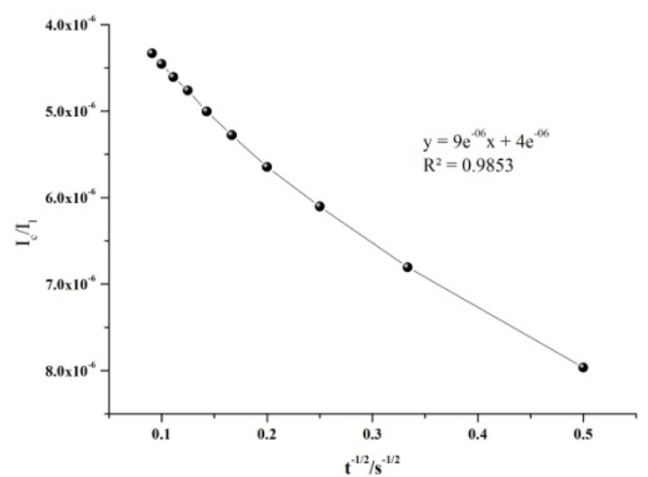

$$
\mathrm{I}=\mathrm{nFAD}{ }^{1 / 2} \mathrm{C}^{-1 / 2} \mathrm{t}^{-1 / 2}
$$

using the slope of I against $\mathrm{t}^{-1 / 2}$. Fig. 6 (right) shows I vs. $\mathrm{t}^{-1 / 2}$ diagram based on which, $D$ was obtained as $1.5 \times 10^{-6} \mathrm{~cm}^{2} \mathrm{~s}^{-1}$ that is similar with other reports [26].

\section{Conclusion}

In this work the electrooxidation of AA on the surface of CoNPsMCPE in alkaline media was studied. The results showed that CoNPsMCPE has good AA oxidation activity in alkaline media and can be a suitable candidate as an AA sensor. The results show that AA electrooxidation on the surface of CoNPsMCPE is irreversible and kinetic properties of the modified electrode are comparable with other cobalt electrodes.

\section{References}

[1] Murray, R.W., A.G. Ewing, and R.A. Durst, Chemically modified electrodes molecular design for electroanalysis. Analytical Chemistry, 1987. 59(5): p. 379A-390A.

[2] Mikysek, T.s., et al., New approaches to the characterization of carbon paste electrodes using the ohmic resistance effect and qualitative carbon paste indexes. Analytical chemistry, 2009. 81(15): p. 6327-6333.

[3] Shabani-Shayeh, J., A. Ehsani, and M. Jafarian, Physioelectrochemical Investigation of Electrocatalytic Activity of Modified Carbon Paste Electrode in Alcohol Oxidation as Anode in Fuel Cell. Journal of the Korean Electrochemical Society, 2014. 17(3): p. 179-186.

[4] Dryhurst, G., et al., Laboratory techniques in electroanalytical chemistry. Marcel Dekker, New York, 1984.

[5] Almeida, C. and B. Giannetti, Electrochemical study of arsenopyrite weathering. Physical Chemistry Chemical Physics, 2003. 5(3): p. 604-610.

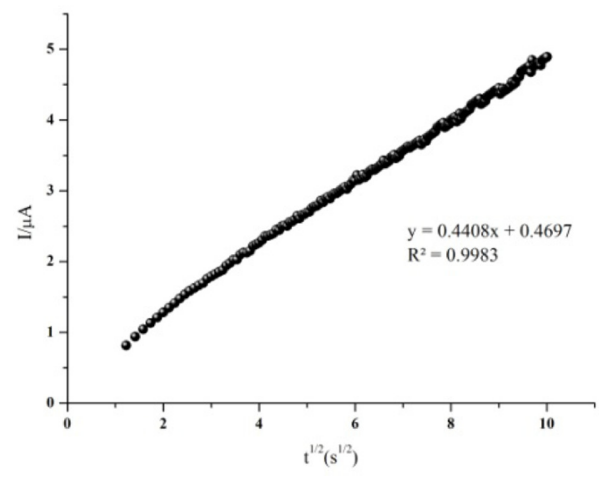

Fig. 6. Dependence of $I_{C} / I_{L}$ on $t^{1 / 2}$ (right) and current on $t^{1 / 2}$ (left) both derived from the chronoamperogams of Fig. 5. 
[6] Yamazaki, S.-i., et al., Electrochemical oxidation of oxalic acid by $R h$ octaethylporphyrin adsorbed on carbon black at low overpotential. Journal of Electroanalytical Chemistry, 2007. 602(1): p. 96-102.

[7] Barbero, C., G.A. Planes, and M.C. Miras, Redox coupled ion exchange in cobalt oxide films. Electrochemistry communications, 2001. 3(3): p. 113116.

[8] Bojinov, V.B., I.P. Panova, and J.-M. Chovelon, Novel blue emitting tetra-and pentamethylpiperidin-4-yloxy-1, 8-naphthalimides as photoinduced electron transfer based sensors for transition metal ions and protons. Sensors and Actuators B: Chemical, 2008. 135(1): p. 172-180.

[9] Hu, P., et al., Highly formaldehyde-sensitive, transitionmetal doped $\mathrm{ZnO}$ nanorods prepared by plasmaenhanced chemical vapor deposition. Sensors and Actuators B: Chemical, 2012. 169: p. 74-80.

[10] Ceken, B., M. Kandaz, and A. Koca, Electrochemical metal-ion sensors based on a novel manganese phthalocyanine complex. Synthetic Metals, 2012. 162(17): p. 1524-1530.

[11] Su, P.-G., L.-G. Lin, and P.-H. Lin, Detection of Cu (II) ion by an electrochemical sensor made of 5, 17-bis (4nitrophenylazo)-25, 26, 27, 28-tetrahydroxycalix [4] arene-electromodified electrode. Sensors and Actuators B: Chemical, 2014. 191: p. 364-370.

[12] Ji, J., et al., Electrochemical sensor based on molecularly imprinted film at Au nanoparticles-carbon nanotubes modified electrode for determination of cholesterol. Biosensors and Bioelectronics, 2015. 66: p. 590-595.

[13] Santos, V.N., et al., Study of a gold electrode modified by trans-[Ru (NH 3) 4 (Ist) SO 4]+ to produce an electrochemical sensor for nitric oxide. Electrochimica Acta, 2011. 56(16): p. 5686-5692.

[14] Nkeng, P., et al., Enhancement of surface areas of Co 3 $\mathrm{O} 4$ and $\mathrm{NiCo} 2 \mathrm{O} 4$ electrocatalysts prepared by spray pyrolysis. Journal of Electroanalytical Chemistry, 1996. 402(1): p. 81-89.

[15] Spinolo, G., S. Ardizzone, and S. Trasatti, Surface characterization of $\mathrm{Co} 3 \mathrm{O} 4$ electrodes prepared by the sol-gel method. Journal of Electroanalytical Chemistry, 1997. 423(1): p. 49-57.

[16] Schumacher, L.C., et al., Semiconducting and electrocatalytic properties of sputtered cobalt oxide films. Electrochimica Acta, 1990. 35(6): p. 975-984.

[17] Heli, H. and H. Yadegari, Nanoflakes of the cobaltous oxide, CoO: Synthesis and characterization. Electrochimica Acta, 2010. 55(6): p. 2139-2148.

[18] Nasrollahzadeh, M., S.M. Sajadi, and M. Khalaj, Green synthesis of copper nanoparticles using aqueous extract of the leaves of Euphorbia esula $L$ and their catalytic activity for ligand-free Ullmann-coupling reaction and reduction of 4-nitrophenol. RSC Advances, 2014. 4(88): p. $47313-47318$

[19] Nasrollahzadeh, M., et al., Green synthesis of $\mathrm{Pd} / \mathrm{Fe} 3 \mathrm{O} 4$ nanoparticles using Euphorbia condylocarpa M. bieb root extract and their catalytic applications as magnetically recoverable and stable recyclable catalysts for the phosphine-free Sonogashira and Suzuki coupling reactions. Journal of Molecular Catalysis A: Chemical, 2015. 396: p. 31-39.

[20] Nasrollahzadeh, M. and M. Maham, RSC Adv, 2014, 4, 19731 RSC;(b) M. Nasrollahzadeh, A. Rostami-Vartouni, A. Ehsani and M. Moghadam. J. Mol. Catal. A: Chem, 2014. 387: p. 123.

[21] Nasrollahzadeh, M., et al., Facile and surfactant-free synthesis of Pd nanoparticles by the extract of the fruits of Piper longum and their catalytic performance for the Sonogashira coupling reaction in water under ligandand copper-free conditions. RSC Advances, 2015. 5(4): p. 2562-2567.

[22] Bruckenstein, S. and M. Shay, Experimental aspects of use of the quartz crystal microbalance in solution. Electrochimica Acta, 1985. 30(10): p. 1295-1300.

[23] Bard, A.J. and L.R. Faulkner, Electrochemical methods: fundamentals and applications. Vol. 2. 1980: Wiley New York.

[24] Karim-Nezhad, G., et al., Electro-oxidation of ascorbic acid catalyzed on cobalt hydroxide-modified glassy carbon electrode. Journal of the Serbian Chemical Society, 2009. 74(5): p. 581-593.

[25] Jing, Z. and X. Lin, Electrocatalytic Oxidation of Formaldehyde on Copper Oxide Nano-crystalline Modified Glassy Carbon Electrode. Chinese Journal of Chemistry, 2010. 28(12): p. 2359-2363.

[26] Roy, P.R., et al., Electrooxidation and Amperometric Detection of Ascorbic Acid at GC Electrode Modified by Electropolymerization of $N, N$-Dimethylaniline. Electroanalysis, 2004. 16(4): p. 289-297. 